\title{
Collective dynamics of glass-forming polymers at intermediate length scales
}

\section{A synergetic combination of neutron scattering, atomistic simulations and theoretical modelling}

\author{
Juan Colmenero ${ }^{1,2,3, a}$, Fernando Alvarez ${ }^{1,2, b}$ and Arantxa Arbe ${ }^{1, \mathrm{c}}$ \\ ${ }^{1}$ Centro de Física de Materiales (CSIC, UPV/EHU) and Materials Physics Center MPC, Paseo Manuel de Lardizabal 5, \\ 20018 San Sebastián, Spain \\ ${ }^{2}$ Departamento de Física de Materiales (UPV/EHU), Apartado 1072, 20080 San Sebastián, Spain \\ ${ }^{3}$ Donostia International Physics Center, Paseo Manuel de Lardizabal 4, 20018 San Sebastián, Spain
}

\begin{abstract}
Motivated by the proposition of a new theoretical ansatz [V.N. Novikov, K.S. Schweizer, A.P. Sokolov, J. Chem. Phys. 138, 164508 (2013)], we have revisited the question of the characterization of the collective response of polyisobutylene at intermediate length scales observed by neutron spin echo (NSE) experiments. The model, generalized for sublinear diffusion -as it is the case of glass-forming polymers- has been successfully applied by using the information on the total self-motions available from MD-simulations properly validated by direct comparison with experimental results. From the fits of the coherent NSE data, the collective time at $Q \rightarrow 0$ has been extracted that agrees very well with compiled results from different experimental techniques directly accessing such relaxation time. We show that a unique temperature dependence governs both, the $Q \rightarrow 0$ and $Q \rightarrow \infty$ asymptotic characteristic times. The generalized model also gives account for the modulation of the apparent activation energy of the collective times with the static structure factor. It mainly results from changes of the short-range order at inter-molecular length scales.
\end{abstract}

\section{Introduction}

Deep understanding of the complex dynamics taking place in glass-forming systems could potentially be gained by exploiting the information provided by the collective response monitored by coherent neutron scattering. For instance, questions like the characterization of the cooperativity or the heterogeneities in the dynamics of these systems could be addressed at the proper length scales. Of particular interest is therefore to cover the crucial mesoscopic regime, i.e., the region of the socalled intermediate length scales. Intermediate length scales denote the regime of lengths larger than the interparticle distances but smaller than the hydrodynamic range. However, this is nowadays still a white area on the relaxation map of the dynamics of glass-forming liquids. The challenge is to characterize from both, an experimental and a theoretical viewpoint, the dynamic structure factor $S(Q, t)$ in the $Q$ (wavevector) range between the first structure factor peak -amorphous haloand the hydrodynamic range.

The collective response in the hydrodynamic regime can be well investigated by quasielastic light scattering at length scales of the order of several $100 \mathrm{~nm}$; other

\footnotetext{
a e-mail: juan.colmenero@ehu.es

b e-mail: fernando.alvarez@ehu.es

c e-mail: a.arbe@ehu.es
}

macroscopic techniques like e.g. dielectric or mechanical spectroscopy also allow covering such a low- $Q$ limit. On the other extreme, neutron scattering has already been used to investigate the dynamic structure factor at the first structure factor peak $Q_{\max }$, reveling thereby the "genuine" $\alpha$-relaxation, in a relatively large number of glassforming systems, and in particular in polymers [1-13]. In fact, the application of neutron scattering techniques to protonated and deuterated samples (addressing thereby the self-motions of hydrogens and the collective response, respectively, see Appendix A) has been key to characterize the dynamics of the structural relaxation at inter- and intra-molecular length scales $[8,14,15]$. Despite the big advantages of this technique - e.g., providing microscopic information with space/time resolution, possibility of selective studies through deuteration labeling, and direct theoretical interpretation of the magnitudes measured in terms of the Van Hove formalism -, it also suffers some limitations which sometimes prevent a clear interpretation of the results (see Appendix A). Therefore, its combination with other techniques, and in particular with computer simulations, can be of utmost help. Let us make some remarks about this combined approach.

Computer simulations in general - i.e., ab-initio methods, classical molecular dynamics simulations, Monte Carlo methods, etc. - are considered to be in between theoretical approaches and experimental tools. However, roughly speaking, there are two different ways of doing 
simulations: (i) the point of view of a theoretician and (ii) that of an experimentalist. In the first case, the focus is on simulating simple systems which capture the essence of a given problem and which can be used to check theories and theoretical concepts. No direct connection with real systems is usually invoked. In the second case, the focus is on mimicking real systems as much as possible. To do that, fully atomistic simulations should be, in principle, the best choice. However, a validation of the simulated system by comparison with experimental results becomes crucial. Once the simulated system is validated -at least for the particular problem we are dealing with- we can take advantage of the simulations, for instance, calculating magnitudes that are not experimentally observable or extending the parameters range beyond the experimental capabilities. By means of this feedback between experiments and simulations we can try to understand a given problem and, of course, we can check theories as well.

Neutron scattering techniques are the right tools to validate fully atomistic MD-simulations of soft materials, in particular in polymer systems. First of all, they cover the relevant length and time scales. Moreover, the classical van Hove formalism allows easily calculating the magnitudes measured by neutron scattering from the simulated atomic trajectories. Different correlation functions can be experimentally addressed (via deuterium labeling) and used for exhaustive check of the reliability of the simulated cell. The methodology employed in the synergetic combination of neutron scattering and fully atomistic MD-simulations to characterize the structure and dynamics of glass-forming polymers at local length scales has proved to provide a highly successful tool. This synergetic combination was explained and illustrated with some representative examples in a recent tutorial review [16].

The aim of the present work is to realize a "collective" effort employing a combination of neutron scattering experimental results and fully atomistic MD-simulations to try to shed some light on the collective dynamics of a glass-forming polymer, polyisobutylene (PIB), in the intermediate length scales regime. This objective is by no means trivial, due to the following difficulties: Experimentally, in this region we must face (i) extremely low intensities (ii) significant contributions of multiple scattering which originates from the the primary scattering at the first structure factor peak and (iii) slow times of the motions and stretched functional forms of the dynamic structure factor. Concerning computer simulations, the intermediate length $/ Q$ regime is even a more difficult range than for neutron scattering. This is because in order to calculate coherent scattering at low $Q$-values of the order of $0.1-0.2 \AA^{-1}$ for a cubic simulated cell, the size of this cell has to be rather large (more than $100 \AA$ side), containing thereby a huge number of atoms. Moreover, for the low- $Q$ values, there are only very few reciprocal vectors complying with the periodic boundary conditions. Usually this leads to unsatisfactory statistics in the case of the calculated collective scattering function. Finally, the lack of a proper theoretical framework to guide the data interpretation constitutes a huge

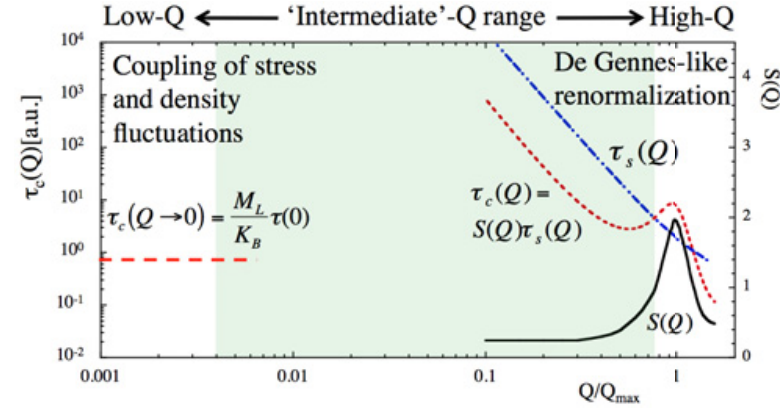

Figure 1. Cartoon of the relaxation map in glass-forming systems: self-correlation times (blue dashed-dotted line) and collective time (red dashed line at low- $Q$ values, red dotted line at high- $Q$ values). The structure factor $S(Q)$ is shown for comparison (black solid line). The functions are plotted against the reduced variable $Q / Q_{\max }$ (wavevector normalized to the position of the first structure factor peak). Shadowed area sketches the intermediate length scales region.

obstacle. For the local and intermolecular level there exist more or less accepted theoretical approaches as for instance the mode coupling theory [17]- or phenomenological ansatzs to relate coherent and selfdynamics -like the deGennes narrowing [18] or the Sköld approximation [19]-. The continuous limit case $Q \rightarrow 0$ has also been theoretically treated [20]. However, up to date there is not any theory or even a statisticalbased model bridging the gap of the intermediate scales. The current situation is somewhat puzzling, as it is schematically illustrated in the cartoon of Fig. 1. If a phenomenological renormalization like that of de Gennes is applied to the characteristic times for self-motions and extrapolated toward intermediate length scales, the collective time would tend to continuously increase in approaching the hydrodynamic regime. However, this would not be compatible with the results commonly found for such range, where the collective characteristic time usually takes values not very different from those observed in the neighborhood of the first structure factor peak.

In this direction, a recent paper [21] has provided a valuable ansatz to solve this controversy. The authors of Ref. [21] have formulated a simple analytical model for the temperature and $Q$-dependent collective density fluctuation relaxation time $\tau_{c}(Q, T)$ in a wide $Q$-range covering both the $S(Q)$ and the intermediate $Q$-region of glass-forming systems. They successfully applied this model to available neutron scattering data corresponding to the ionic system $\mathrm{Ca}-\mathrm{K}-\mathrm{NO}_{3}(\mathrm{CKN})$. However, in order to apply this approach to glass-forming polymers, it needs to be modified to take into account the particularities of the local and diffusive motions in polymer melts. With these ideas in mind, in Ref. [22] we developed a generalization of that model that applies for the case of anomalous diffusion. Here, we show in detail how this generalization is formulated and applied to the case of PIB, for which the best experimental data on collective relaxation exist up to date [6]. To achieve this, we make use of the information provided by properly validated MD-simulations. In addition, we compile in a single relaxation map data for collective dynamics 
of PIB deduced from the application of the proposed ansatz and from different techniques including dielectric spectroscopy [3], light scattering [23] and mechanical measurements [24], obtaining a consistent picture of the collective response.

\section{Experimental results}

In this work we discuss on neutron spin echo (NSE) results previously published in Ref. [6]. The experiments on collective dynamics were performed on a fully deuterated sample dPIB (see details for synthesis and purification in Ref. [25]) displaying a glass-transition temperature $T_{g}=$ $205 \mathrm{~K}$. The molecular weight was $M_{n}=72000 \mathrm{~g} / \mathrm{mol}$ and the polydispersity $M_{w} / M_{n}=1.05$. The sample (thickness: $2 \mathrm{~mm}$ ) was placed on a flat aluminum container yielding a perpendicular transmission of 0.85 (measured with a wavelength of $6 \AA$ ). Just for validation purposes we will also consider the NSE results on a fully protonated sample hPIB reported on the same article.

The NSE instrument employed was IN11C at the Institut Laue Langevin (Grenoble, France). Two incident wavelengths ( 6 and $10 \AA)$ were used, covering a $Q$-range $0.2 \leq Q \leq 1.6 \AA^{-1}$-which includes the region of the first peak $Q_{\max }=1 \AA^{-1}-$ in a maximum $t$-range $8 \mathrm{ps} \leq t \leq 5.5 \mathrm{~ns}$. Three temperatures $(335,365$ and $390 \mathrm{~K})$ were investigated. The background-corrected data were divided by the resolution function revealing finally the normalized intermediate dynamic structure factors $N S E(Q, t)$ (see Appendix A). Further details on the experimental setup and data reduction can be found in Ref. [6]. Figure 2 shows some representative examples of the results obtained for the deuterated sample (upper panel) and the protonated sample (lower panel). We recall that they are dominated by the collective dynamic structure factor and the incoherent scattering function of all hydrogens in the sample respectively (see Appendix A).

The experimental data in Ref. [6] were described in terms of Kohlrausch-Williams-Watts (KWW) or stretched exponential functions:

$$
\Phi(Q, t) \propto \exp \left[-\left(\frac{t}{\tau_{w}(Q)}\right)^{\beta}\right] .
$$

Due to the limited dynamic window explored and the experimental uncertainties involved, a precise determination of the stretching exponent $\beta$ as function of $Q$ and temperature is not possible. Therefore, a fixed value of 0.55 was assumed to describe all the NSE results. For the three temperatures investigated, the average values obtained for the collective times $\left\langle\tau_{c}(Q)\right\rangle$ of PIB - i.e., deduced from the NSE results on the deuterated sample - are shown in Fig. 3. We recall that the average time of a given correlation function $\Phi(t)$ is defined as $\langle\tau\rangle=\int_{0}^{\infty} \Phi(t) d t$ and in the case of a stretched exponential functional form (Eq. 1), it is given by $\langle\tau\rangle=\frac{\Gamma(1 / \beta)}{\beta} \tau_{w}$.

Experimental information on the collective times of PIB in the low $Q$-limit $\tau_{c}(Q \rightarrow 0)$ can be extracted from the literature. From measurements on the elastic modulus at $298 \mathrm{~K}$ [24] a value of $64 \mathrm{~ns}$ can be deduced for this time [6]. This value is represented as a grey horizontal

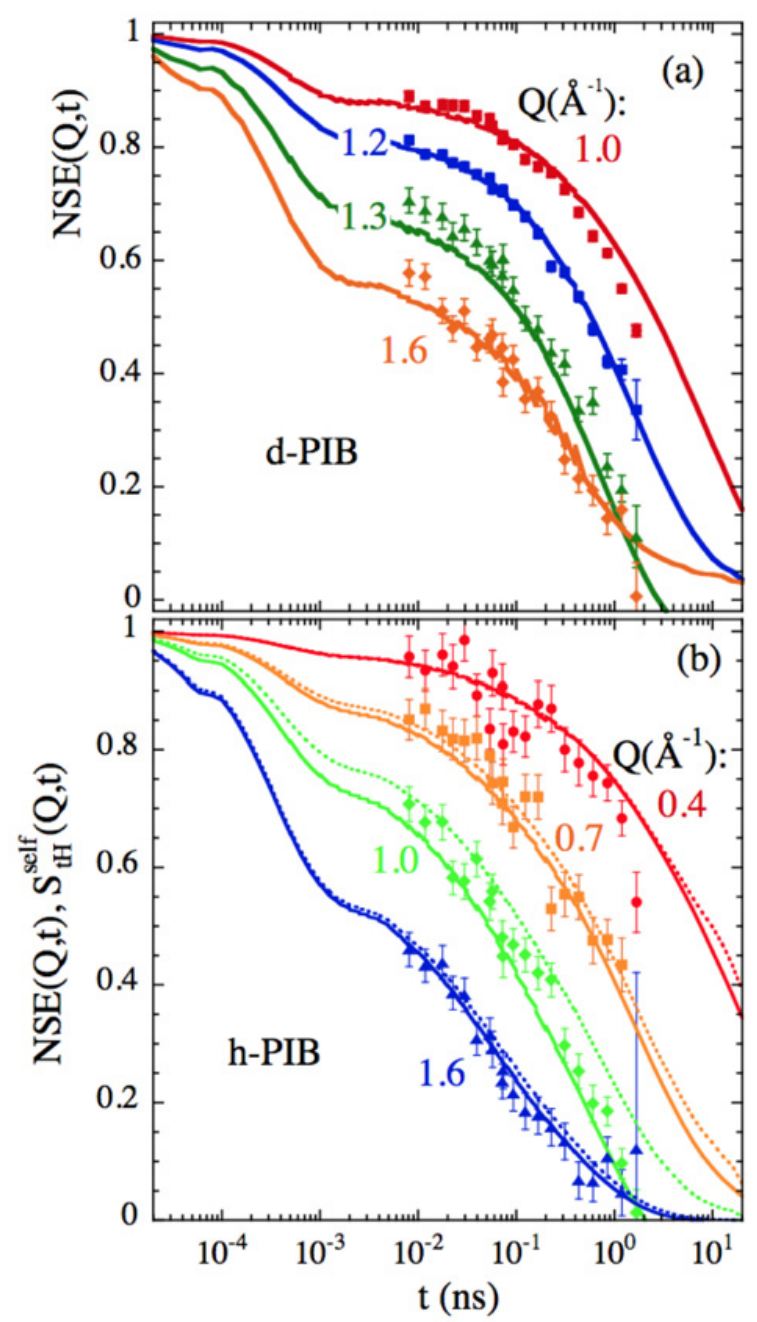

Figure 2. NSE results measured (symbols) [6] and calculated from the simulations (solid lines) on a deuterated PIB sample (a) and a protonated PIB sample (b) at $T=365 \mathrm{~K}$ and the $Q$-values indicated. Bandpass corrections have been applied to the experimental data (see, e.g. [26]). The value considered for the band pass time was $0.2 \mathrm{ps}$. For comparison, the $S_{t H}^{\text {self }}(Q, t)$ calculated for all hydrogens is shown by the dotted lines in (b).

arrow in Fig. 3. Furthermore, results from Brillouin light scattering [23] point to a value of $64 \mathrm{ps}$ for the collective time [6] at $473 \mathrm{~K}$, depicted as a violet horizontal arrow in Fig. 3.

\section{Modelling}

The model used to describe the collective response at intermediate length scales consists of an interpolation formula that embeds the mesoscopic (non-diffusive) and the high- $Q$ (diffusive) limits of the collective times in an analytical expression as proposed by Novikov et al. [21]:

$$
\frac{1}{\tau_{c}(Q)}=\frac{1}{\tau_{c}(Q \rightarrow 0)} e^{-Q^{2} \xi_{c}^{2}}+\frac{1}{\tau_{c}^{D}(Q)} .
$$

The first term of this expression contains the inverse of a non-diffusive ( $Q$-independent) relaxation time $\tau_{c}(Q \rightarrow$ $0)=\frac{M_{L}}{K_{B}} \tau_{\alpha}(0)$. Here $K_{B}$ is the bulk modulus and 


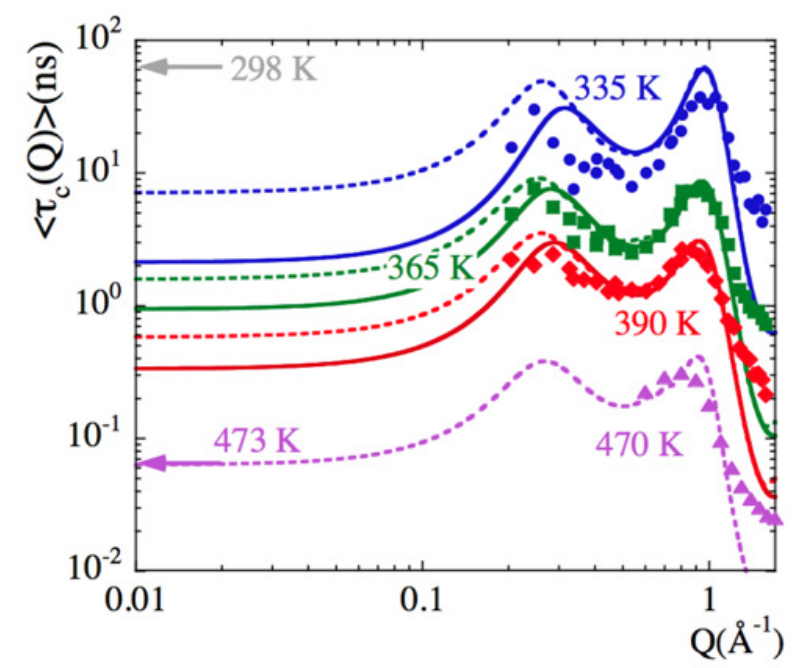

Figure 3. Momentum transfer dependence of the average characteristic times for collective motions obtained from NSE measurements on deuterated PIB [6] at 335 (circles) 365 (squares) and $390 \mathrm{~K}$ (diamonds). Triangles show the results obtained from the MD-simulations at $470 \mathrm{~K}$. Arrows mark the values deduced for $\tau_{c}(Q \rightarrow 0)$ at 298 and $473 \mathrm{~K}$ from the literature. Lines represent the description by Eq. (8): solid lines were obtained by fitting the value of $\tau_{c}(Q \rightarrow 0)$, and dotted lines by fixing $\tau_{c}(Q \rightarrow 0)$ to the values corresponding to the solid line in Fig. 8.

$M_{L} \approx K_{B}+(4 / 3) G$ the longitudinal elastic modulus, where $G$ is the elastic shear modulus. The time $\tau_{\alpha}(0)$ has the meaning of an $\alpha$ (structural) relaxation time associated with a typical $Q \sim 0$ correlation function. The authors of Ref. [21] identify it with the time corresponding for instance to a dielectric or viscoelastic response. This time should reflect the viscoelastic coupling of stress and density fluctuations on scales long enough compared to atomic dimensions, but not yet in the hydrodynamic limit. The contribution of this first term to the total collective time is affected by a Gaussian cutoff factor $e^{-Q^{2} \xi_{c}^{2}}$ to ensure that it is present only on length scales beyond a characteristic length $\xi_{c}$, which is assumed to be $\xi_{c} \sim$ $2 \pi / Q_{\max }$.

To model the diffusive time $\tau_{c}^{D}(Q)$, we generalized the expression given in Ref. [21] to the case of sublinear diffusion. As in Ref. [22], we considered the ansatz proposed by Sköld [19]. This approach consists of the renormalization of $Q$ by the structure factor:

$$
\frac{S(Q, t)}{S(Q)}=S^{s e l f}\left(\frac{Q}{\sqrt{S(Q)}}, t\right)
$$

For simple diffusion Eq. (3) naturally reproduces the de Gennes narrowing. In order to obtain an analytical expression for $\tau_{c}^{D}(Q)$ based in the Sköld's approximation, we first need an analytical description of the selfcorrelation function (this is naturally a KWW function) and the associated incoherent characteristic time. In the Gaussian approximation -which is usually well fulfilled for polymers at $Q$-values below and around the first structure factor peak- the $Q$-dependence of the characteristic time in a KWW self-correlation function is given by [27]

$$
\tau_{s} \propto Q^{-2 / \beta_{s}} .
$$

Usually the values of the stretching exponent $\beta_{s}$ are hardly $Q$-dependent and then the normalized dynamic structure factor after Eq. (3) is a KWW function with the same $\beta_{S^{-}}$ parameter and $\tau_{c}^{D}(Q) \propto S(Q)^{1 / \beta_{s}} Q^{-2 / \beta_{s}}$, i.e.,

$$
\tau_{c}^{D}(Q)=S(Q)^{1 / \beta_{s}} \tau_{s}(Q) .
$$

The deviations from Gaussian behavior usually observed at high $Q$-values in the characteristic times for self-motions in glass-forming polymers can be well accounted for by the so-called anomalous jump diffusion model [28,29]. As in the case of the simple jump-diffusion model [30,31], this approach also considers a distribution of jump distances $f_{o}(\xi)=\frac{\xi}{\xi_{0}^{2}} \exp \left(-\frac{\xi}{\xi_{o}}\right)$. Here, $\xi_{o}$ is the preferred jump distance. In this framework, the incoherent relaxation time $\tau_{s}(Q)$ can be expressed as

$$
\tau_{s}(Q)=\tau_{o}\left[1+\frac{1}{Q^{2} \xi_{o}^{2}}\right]^{\frac{1}{\beta_{s}}} .
$$

Combining Eqs. (5) and (6) we obtain for the diffusive collective time:

$$
\tau_{c}^{D}(Q)=S(Q)^{\frac{1}{\beta_{s}}} \tau_{o}\left[1+\frac{1}{Q^{2} \xi_{o}^{2}}\right]^{\frac{1}{\beta_{s}}} .
$$

The total collective time can then be described by Eq. (2) combined with Eq. (7):

$$
\frac{1}{\tau_{c}(Q)}=\frac{1}{\tau_{c}(Q \rightarrow 0)} e^{-Q^{2} \xi_{c}^{2}}+S(Q)^{-\frac{1}{\beta_{s}}} \tau_{o}^{-1}\left[1+\frac{1}{Q^{2} \xi_{o}^{2}}\right]^{-\frac{1}{\beta_{s}}} \text {. }
$$

This total expression reduces to that proposed in Ref. [22] for the case $\beta_{s}=1$ (normal jump diffusion).

Applying the proposed ansatz with only experimental information at hand is unfortunately not possible, since the self-correlation time $\tau_{s}$ involved in this model cannot be measured -we recall that neutrons are not sensitive to the self-motions of carbon atoms-. At this point is where the use of MD-simulations becomes imperative. It is worth emphasizing that what we need now is information about the self-motions, i.e., we have not the problems involved in the calculation of the coherent scattering functions at low $Q$.

\section{Molecular dynamics simulations}

The atomistic simulations were carried out by means of the COMPASS forcefield. The cubic simulation cell contained 20 PIB chains of 70 monomers each (total number of atoms $N=16840$ ). Initially, by means of NPT dynamic runs (i.e., keeping constant $N$, pressure and temperature) values of the density close to the experimental ones at the different temperatures simulated $(320,335,365,390$ and $470 \mathrm{~K})$ were obtained. The dimension of the cell in such conditions was of the order of $54 \AA$ side. After that, successive NVT equilibration runs (i.e., keeping constant $N$, volume and temperature) were carried out followed by the production of dynamic runs. The simulation time 


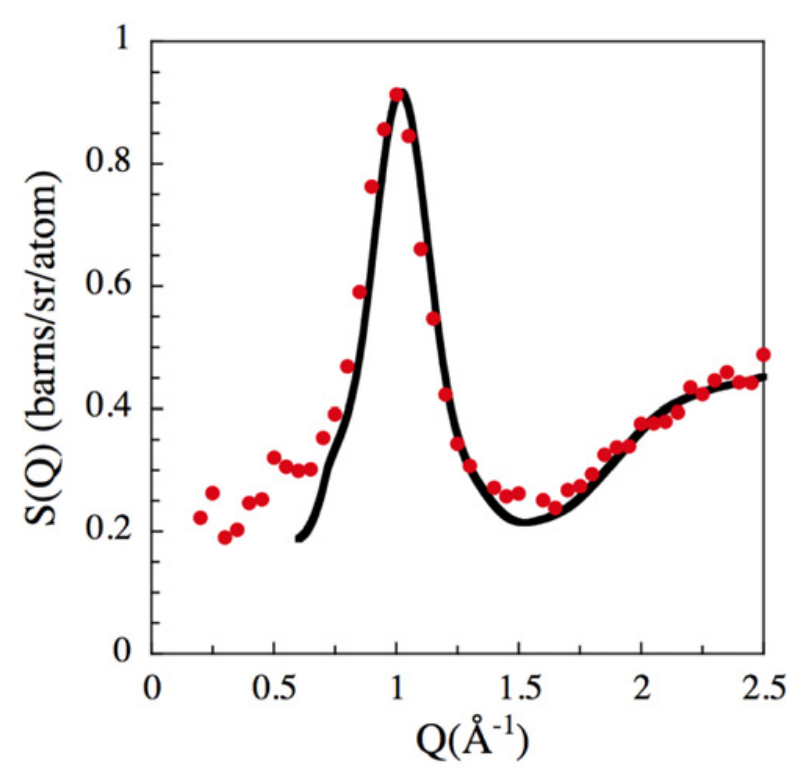

Figure 4. Comparison of the static structure factor of PIB experimentally determined on a perdeuterated sample by tripleaxis (filled circles) [6] and computed (line) at $320 \mathrm{~K}$.

was extended until $100 \mathrm{~ns}$. More simulation details can be found in Ref. [32].

Figure 2 demonstrates the reliability of the simulation cell by directly comparing the NSE results measured (symbols) and calculated from the simulations (lines) for the deuterated sample (upper panel) and the protonated sample (lower panel). The agreement is perfect, in particular taking into account that there is not any timeshift between simulated and measured data. The validation of the structural features of the cell was also performed by direct comparison with experimentally obtained data. This is shown in Fig. 4.

Once the cell is validated, we may exploit the information contained in the atomic trajectories and calculate functions which are not accessible experimentally or check the correctness of usually assumed hypotheses in the experimental data evaluation. For instance, we have included in Fig. $2 b$ the intermediate incoherent scattering function calculated for all the hydrogen atoms (total hydrogens, $\mathrm{tH}$ ) in the simulated cell. The good agreement between the NSE result and this function corroborates the assumption that the measurements are dominated by the hydrogens' self-motions $S_{t H}^{\text {self }}(Q, t)$. The coherent contribution to the NSE signal is most evident at $Q \approx$ $1 \AA^{-1}$, coinciding with the position of the maximum of the structure factor $Q_{\max }$, and leads to an apparently faster decay of the function. Simulations also allow distinguishing the different atomic species in the system. For a $Q$-value of $1.5 \AA^{-1}$ and $T=335 \mathrm{~K}$, Fig. 5 shows the intermediate incoherent scattering function of the different kinds of atoms in PIB: main-chain carbons (cC), mainchain hydrogens $(\mathrm{cH})$, methyl-group carbons $(\mathrm{mC})$ and methyl-group hydrogens $(\mathrm{mH})$ (see inset in Fig. 5 for the definitions). Figure 5 reveals that: (i) for any type of atom the scattering function decays in two main steps, before and after $\approx 1 \mathrm{ps}$; (ii) the atomic motions in PIB are very

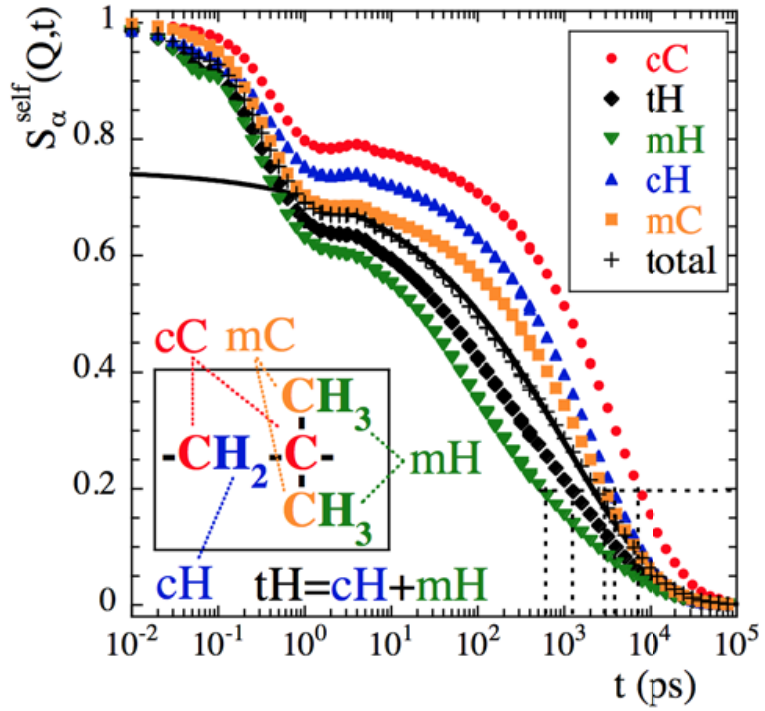

Figure 5. Intermediate scattering function corresponding to the different atomic species of PIB. Dotted lines illustrate the location of the characteristic time corresponding to an $80 \%$ decay of the function. Crosses correspond to the total self-correlation function (all atoms equally weighted). The line is a KWW fit of this function above $2 \mathrm{ps}$.

heterogeneous. Defining a global characteristic time $\tau_{0.2}$ as that at which the function reaches the value of 0.2 (see dotted line in the figure), at $335 \mathrm{~K}$ we find a difference of more than one order of magnitude between $\tau_{0.2}^{m H}$ and $\tau_{0.2}^{c C}$. (iii) the decay at short times is more pronounced for methyl-group atoms than for main-chain atoms, and (iv) the stretching of the second decay is much stronger for $\mathrm{mH}$ than for the other atoms in the system. As expected, the behavior of $\mathrm{tH}$ is very close to that of $\mathrm{mH}-6$ out of the 8 hydrogens in PIB are located in the methyl groups. Finally, we have also calculated the function which is relevant for the application of the proposed model: that corresponding to the self-motions of all atoms in the system ("total"). As can be seen in the figure, a stretched exponential (Eq. (1)) provides an excellent description of the decay of this correlation function for times above $\approx 2 \mathrm{ps}$.

From such fits we have obtained the values of the stretching exponent $\beta_{s}$ and the self-correlation time $\tau_{s}$ of $S_{\text {total }}^{\text {self }}(Q, t)$ for all $Q$ s and temperatures investigated. The exponent $\beta_{s}$ increased with temperature: $0.42(320 \mathrm{~K})$, $0.44(335 \mathrm{~K}), 0.47(365 \mathrm{~K}), 0.49$ (390 K) and 0.53 (470 K). The obtained values of the average characteristic time for self motion $\left\langle\tau_{s}\right\rangle$ are represented in Fig. 6.

\section{Fitting the model}

The anomalous jump diffusion model [Eq. (6)] describes very nicely the average self-characteristic times of PIB, as can be seen in Fig. 6. The value obtained for the most probable jump length $\xi_{o}$ is independent of temperature within the uncertainties and in the fits shown in this figure it has been fixed to $\xi_{o}=0.6 \AA$. The average value of the prefactor $\tau_{o}$, that represents the time an atom stays vibrating in an equilibrium position between two jumps, 


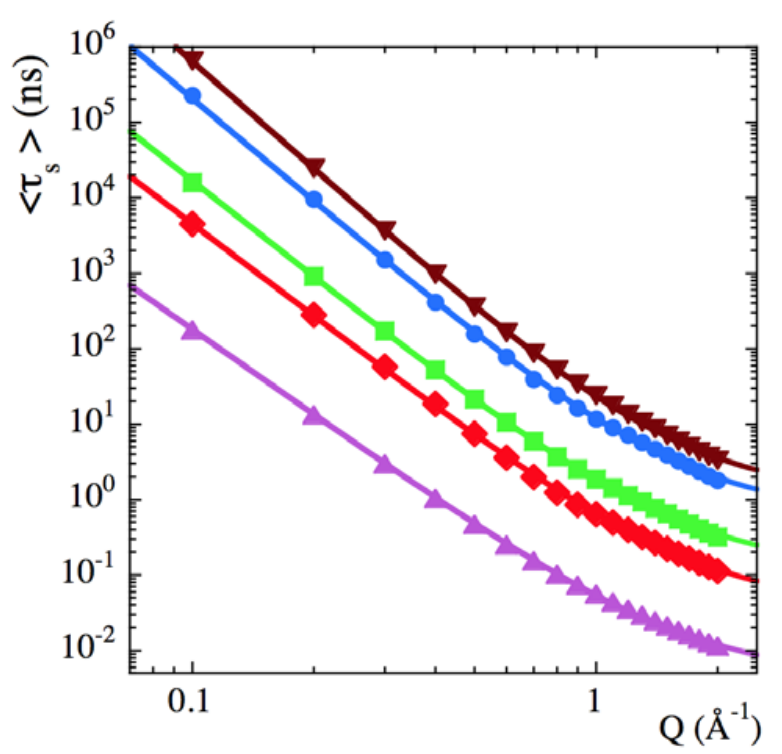

Figure 6. Momentum transfer dependence of the average characteristic times for self motions obtained from MD simulations at 320 (down triangles) 335 (circles) 365 (squares) $390 \mathrm{~K}$ (diamonds) and $470 \mathrm{~K}$ (up triangles). Solid lines correspond to the fits by the anomalous jump diffusion model (Eq. (6)).

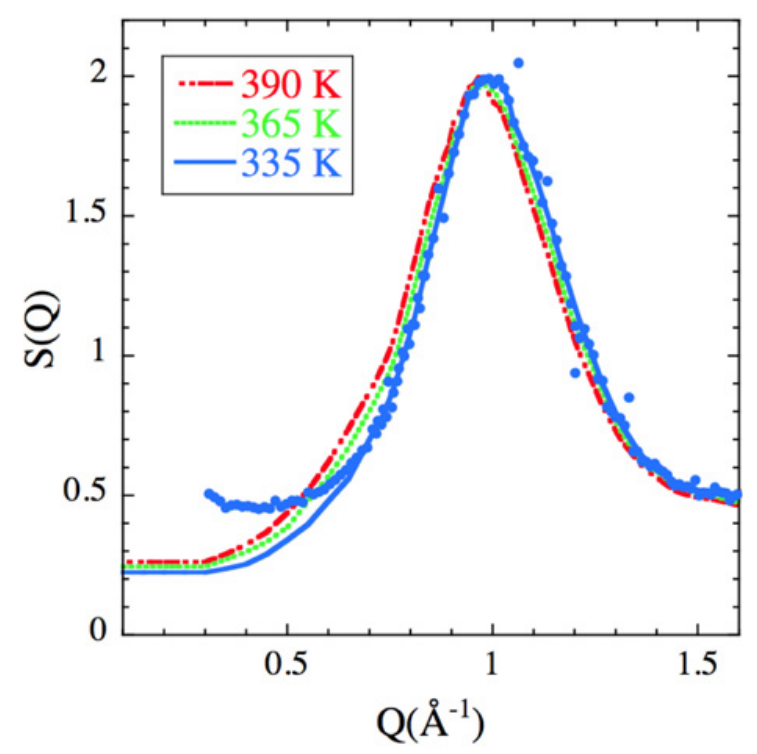

Figure 7. Static structure factor built starting from the NSE data from Ref. [6]. The curves have been obtained by smoothing the experimental results (see as an example the solid circles corresponding to the experimental $335 \mathrm{~K}$ data) and imposing the corresponding theoretical asymptotic low- $Q$ value [6]. We note that the low- $Q$ range is particularly sensitive to multiple scattering events.

has been plotted as function of temperature in Fig. 8 (solid circles).

Once the parameters characterizing the self-motions $\left[\beta_{s}, \tau_{o}\right.$ and $\left.\xi_{o}\right]$ were determined, we fitted Eq. (8) to the experimentally obtained collective times. Using the experimentally determined static structure factor (see Fig. 7), we could fix all the parameters involved in the

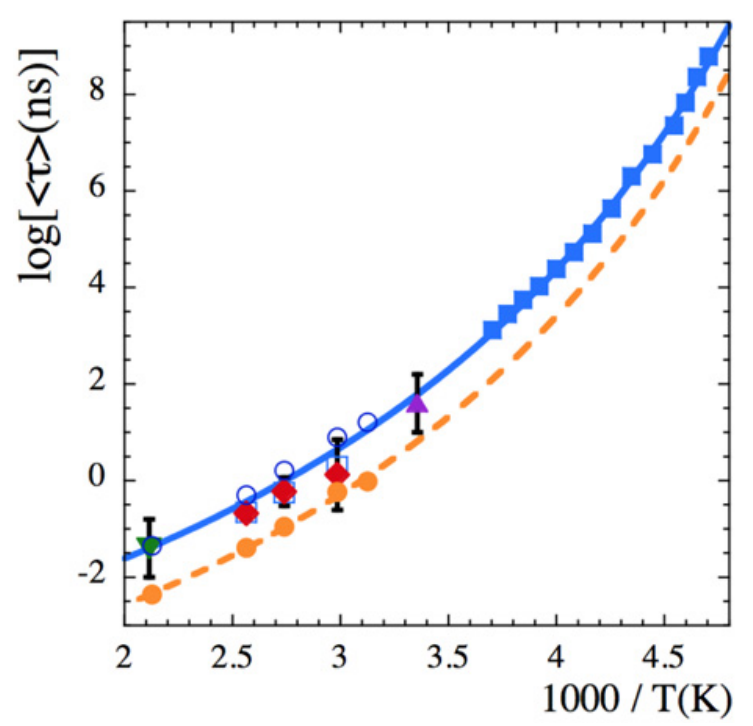

Figure 8. Inverse temperature dependence of the average characteristic times deduced for $\tau_{\alpha}(0)$ from (i) the descriptions of $\left\langle\tau_{c}\right\rangle$ in Fig. 3 (diamonds); (ii) dielectric spectroscopy [3] (filled squares); (iii) elastic modulus [24] (solid up-triangle); (iv) Brillouin light scattering [23] (solid down-triangle). Empty squares represent the results obtained from NSE measurements [6] for the self-motions of hydrogens at $Q=$ $1.0 \AA^{-1}$ and empty circles their simulated counterparts. The elementary average characteristic times for anomalous jump diffusion are also included (solid circles). Lines are VogelFulcher fits with the same $B$ and $T_{o}$ parameters (see text).

description of the diffusive contribution. For the cutoff factor of the low- $Q$ contribution $e^{-Q^{2} \xi_{c}^{2}}$ we imposed the suggested value $\xi_{c}=2 \pi / Q_{\max }$ with $Q_{\max }=1 \AA^{-1}$ as determined from $S(Q)$. Thus, the only free parameter was the characteristic time $\tau_{c}(Q \rightarrow 0)$. The obtained descriptions (solid lines in Fig. 3) are excellent, taking into account that everything is fixed except the value of this parameter. We may comment on the apparent systematic deviations observable for $335 \mathrm{~K}$. The reason could be the higher uncertainties involved in the determination of the experimental characteristic times and the shape of the relaxation function in the low temperature range. All the NSE results were described assuming $\beta=0.55$, but from the simulations a decrease from 0.55 at $390 \mathrm{~K}$ to 0.5 at $335 \mathrm{~K}$ is found [32]. At $335 \mathrm{~K}$, the NSE window is sensitive to the faster part of the decay of the dynamic structure factor. Using $\beta=0.55$ instead of 0.5 , shorter times than the actual ones are obtained and, in addition, the factor $\frac{\Gamma(1 / \beta)}{\beta}$ used for the conversion to average times is smaller, leading to overall faster collective times deduced from the NSE data.

From the values obtained for $\tau_{c}(Q \rightarrow 0)$ and considering $M_{L} / K_{B}, \approx, 1.6$ for PIB -as deduced from Ref. [33]- we calculated the values of the time $\tau_{\alpha}(0)$. The results are displayed in Fig. 8 as red diamonds. In this figure we have also included the above mentioned results deduced for this characteristic time from mechanical and light scattering experiments at room temperature and $473 \mathrm{~K}$ respectively (filled triangles), and from dielectric spectroscopy reported in Ref. [3] (filled squares). As can be appreciated, all these data nicely combine, 
within the uncertainties. This agreement strongly supports the interpretation proposed in Ref. [21] for $\tau_{\alpha}(0)$ deduced from the fits of $\tau_{c}$ as a characteristic time for structural relaxation at $Q \rightarrow 0$. Conversely, it has been reported $[34,35]$ that the dielectric time scale in glass-forming polymers usually coincides with the incoherent times obtained in protonated samples at $Q \sim 1 \AA^{-1}$. Although the reason for this agreement is not yet understood, it is an experimental fact. Figure 8 also includes the experimental values of the incoherent relaxation times corresponding to the hydrogen motions at the $Q$-value of $1 \AA^{-1}$ [6] (empty squares) and their simulated counterparts (empty circles). The empirical finding also applies in the case of PIB, and the good agreement among the compiled results in Fig. 8 illustrates the consistency of the proposed framework. It is worth emphasizing that a single Vogel-Fulcher law $\left\langle\tau_{\alpha}(T)\right\rangle=\left\langle\tau_{\infty}^{\alpha}\right\rangle \exp \left(\frac{B}{T-T_{o}}\right)$ with $\left\langle\tau_{\infty}^{\alpha}\right\rangle=4.6 \times 10^{-14} \mathrm{~s}, B=2277 \mathrm{~K}$ and $T_{o}=136.5 \mathrm{~K}$ (solid line in the figure) describes the whole set of data with high accuracy. Another remarkable observation is that the average characteristic time deduced for the elementary jumps involved in the diffusional process $\tau_{o}$ follows just the same temperature dependence: a VogelFulcher law with the same $B$ and $T_{o}$ parameters as for $\tau_{\alpha}(T)$ perfectly describes these data, with an about ten-fold faster prefactor.

Considering then that the characteristic time $\tau_{\alpha}(Q \rightarrow$ 0 ) can be well parametrized by the above given VogelFulcher equation, in a further refinement step we have imposed such theoretical values in the fits of Eq. (8) to the experimental NSE data. Thus, no fitting parameter was included in this description. The resulting curves are shown as dotted lines in Fig. 3. The agreement is still very good. Finally, we considered the simulation results at $470 \mathrm{~K}$, for which direct information about the value of $\tau_{\alpha}(Q \rightarrow 0)$ exists [23]. Figure 3 displays with the triangles the average collective time deduced from our simulations. We constructed the predicted model curve for $T=$ $470 \mathrm{~K}$ following exactly the same procedure as described above for the experimental data, and now considering the structure factor obtained from the simulations for this temperature. Though the $Q$-range covered by the simulations for the calculation of the collective response is quite narrow, the model describes very well the results -we recall that no fit parameter is involved in this description.

Last, we note that the proposed ansatz also reproduces the a priori unexpected $Q$-dependence observed for the activation energy $E_{a}$ of the collective times by NSE [6]. The points in Fig. 9 represent the experimentally determined apparent activation energy in the temperature range investigated $(335 \leq T \leq 390 \mathrm{~K})$. This parameter shows a kind of modulation with the static structure factor. We can calculate the predicted $Q$-dependence of the effective activation energy, $E_{a}(Q)$, in the explored temperature range. Given a pair of temperatures $T_{i}$ and $T_{j}$, the apparent activation energy of the collective time in the range $T_{i} \leq T \leq T_{j}$ is:

$$
E_{a}^{i j}=K_{B}\left(\frac{T_{i} T_{j}}{T_{j}-T_{i}}\right) \ln \left[\frac{\left\langle\tau_{c}\left(Q, T_{i}\right)\right\rangle}{\left\langle\tau_{c}\left(Q, T_{j}\right)\right\rangle}\right]
$$

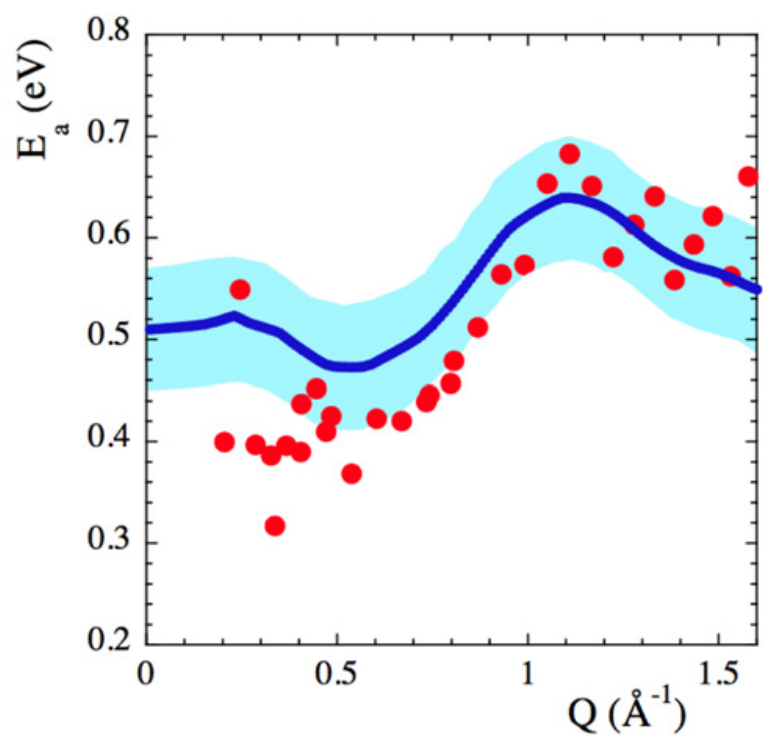

Figure 9. Momentum transfer dependence of the activation energy $E_{a}$ of the collective characteristic times of PIB in the temperature interval $335 \leq T \leq 390 \mathrm{~K}$. Points correspond to the experimental results reported in Ref. [6] and the continuous line to the model results. The shadowed area represents the uncertainty level (see the text).

We have applied this expression to the results of the $\tau_{c}(Q, T)$ predicted by the model (i.e., by imposing all the parameters as above explained) at the three temperatures experimentally investigated. The average value of $E_{a}(Q)$ obtained in this way is shown in Fig. 9. The shadowed area has been calculated taking into account the different values of $E_{a}(Q)$ obtained with the different pairs of temperatures and illustrates the level of uncertainty of this procedure. As can be seen in the figure, the experimental behavior is nicely reproduced, within the uncertainties.

We note that this $E_{a}(Q)$ curve shows a smoother behavior in the low- $Q$ range than that obtained in Ref. [22], which below $Q \approx 0.3 \AA^{-1}$ displayed a drop toward the value $0.4 \mathrm{eV}$. This is because the values for $\tau_{\alpha}$ used in the calculation of $E_{a}(Q)$ in Ref. [22] were those directly obtained from the fits of the experimental $\tau_{c}(Q, T)$ to Eq. (8), and here we have imposed the theoretical values of the Vogel Fulcher law describing the whole set of compiled $\tau_{\alpha}$ data (see Fig. 8). Therefore, the $Q \rightarrow 0$ asymptotic limit of $E_{a}(Q)$ in that case was the apparent activation energy of the three points determined from the fit of the NSE data -subjected to a relatively high degree of uncertaintyand now is the same apparent activation energy as that governing the high- $Q$ limit -note that the same VogelFulcher law (besides the prefactor) described $\tau_{\alpha}(T)$ and the asymptotic $Q \rightarrow \infty$ limit of the self-diffusive time $\tau_{o}$. We note that in the $Q \rightarrow \infty$ limit, collective and selfcorrelation functions coincide; therefore, we observe a unique temperature dependence for both, the $Q \rightarrow 0$ and the $Q \rightarrow \infty$ asymptotic values of the collective times.

Conversely, within the theoretical framework here proposed we may ask what is the origin of the modulation of $E_{a}(Q)$ with the structure factor close to its first peak. It is easy to realize [32] that the collective times $\tau_{c}(Q, T)$ are dominated by the diffusive component $\tau_{c}^{D}(Q, T)$ down 
to $Q$-values of about $0.3 \AA^{-1}$ (where the total collective time reaches a maximum, see Fig. 3). Thus, the activation energy in the range $Q \geq 0.3 \AA^{-1}$ is determined by the temperature dependence of $\tau_{c}^{D}(Q, T)$. From Eq. (7) we can deduce that this modulation has to be due to the variation of the term $S(Q)^{1 / \beta_{s}}$ with temperature, i.e., ultimately to the thermal changes of the structure factor (see Fig. 7). Though apparently weak, the evolution of $S(Q)$ in the intermolecular range -amplified by the $1 / \beta_{s^{-}}$ exponent, which also subtly changes with temperatureis enough to produce sensitive variations in the apparent activation energy of the collective times. A thorough discussion on the origin of the correlations giving rise to the first maximum of the structure factor peak in PIB and its thermal behavior based on the MD-simulations here presented can be found in Ref. [32].

\section{Conclusions}

The ansatz proposed to describe the behavior of the collective response at intermediate length scales in glassforming systems showing anomalous diffusive behavior has been successfully applied to the NSE experimental results of polyisobutylene. To realize this application, the input of information on the self-atomic motions provided by fully atomistic MD-simulations has been indispensable.

We have found a very good agreement between the values of the $\alpha$-relaxation characteristic times associated with a $Q \approx 0$ correlation function obtained through different experimental techniques, namely dielectric spectroscopy, mechanical relaxation and Brillouin light scattering. The values deduced for this parameter from the application of the proposed ansatz to the NSE results also agree very well with that set of data. The same happens with the characteristic times for self-motions of the total hydrogens at $Q=1 \AA^{-1}$-an empirical finding that is corroborated for the case of PIB-. All these times can be very well described by a Vogel-Fulcher law which -shifted by a factor $\approx 0.1-$ also fits the characteristic time governing the discrete jumps underlying the subdiffusive process observed in the high- $Q$ regime. This finding would suggest this jumping mechanism as the elementary process ultimately behind the response of the material at different length scales. Finally, we have shown that the proposed model -with all parameters fixedreproduces well the $Q$-dependence of the activation energy experimentally observed for PIB. The apparent modulation of this parameter with the structure factor results from the temperature dependence of the structural features at intermolecular length scales.

We thank support from the projects IT-654-13 (GV) and MAT2012-31088.

\section{Appendix A: Magnitudes measured in neutron scattering experiments}

Neutrons interact with the atomic nuclei [36-38]. The scattering length -parameter determining the strength of this interaction- depends on the isotope $\alpha$ considered $(\alpha: \mathrm{H}, \mathrm{D}, \mathrm{C}, \mathrm{O}, \ldots)$ and the relative orientation of the neutron-nuclear spin pairs. Table 1 shows the mean values of $b_{\alpha}$ for the isotopes commonly present in soft materials.

The double differential scattering cross section $\partial^{2} \sigma / \partial \Omega \partial \hbar \omega$ usually measured in a neutron scattering experiment is the number of neutrons scattered into a solid angle comprised between $\Omega$ and $\Omega+d \Omega$ and which have experienced a change in energy $\hbar \omega$, with respect to the total number of incident neutrons [36]. The difference between the wavevectors of the scattered $(\vec{k})$ and incident $\left(\vec{k}_{o}\right)$ neutron determines the momentum transfer $\vec{Q}$, which modulus is given by $Q=2 k_{o} \sin (\theta / 2)$ $\left(\theta\right.$ : scattering angle). The interpretation of $\partial^{2} \sigma / \partial \Omega \partial \hbar \omega$ is straightforward in terms of the correlation functions defined in the van Hove (1954) formalism, as shown in the scheme of Fig. 10. There, the indexes $\alpha$ and $\beta$ run over all the possible kinds of isotopes in the sample $(\alpha, \beta: \mathrm{H}, \mathrm{D}, \mathrm{C}$, $\mathrm{O}, \ldots .$.$) . In \partial^{2} \sigma / \partial \Omega \partial \hbar \omega$ we can identify a coherent and an incoherent contribution. The latter arises from the random distribution of the deviations of the scattering lengths from their mean value, $\overline{\Delta b_{\alpha}^{2}}=\overline{b_{\alpha}^{2}}-{\overline{b_{\alpha}}}^{2}$. The features $(\vec{Q}$ and $\omega$-dependencies) of both contributions are determined by the corresponding scattering functions $\left[S_{\alpha \beta}(\vec{Q}, \omega)\right.$ involving nuclei of kinds $\alpha$ and $\beta$, and $S_{\alpha}^{i n c}(\vec{Q}, \omega)$ involving nuclei of kind $\alpha$ ]. These are related, via Fourier transformation, with the intermediate scattering functions $\left[S_{\alpha \beta}(\vec{Q}, t)\right.$ and $\left.S_{\alpha}^{\text {self }}(\vec{Q}, t)\right]$ and the van Hove correlation functions $\left[G_{\alpha \beta}(\vec{r}, t)\right.$ and its self-part $\left.G_{\alpha}^{\text {self }}(\vec{r}, t)\right]$. In the classical limit, $G_{\alpha \beta}(\vec{r}, t)$ can be written as:

$$
G_{\alpha \beta}(\vec{r}, t)=\left\langle\frac{1}{N} \sum_{i \alpha, j \beta}^{N_{\alpha}, N_{\beta}} \delta\left\{\vec{r}-\left[\vec{r}_{i \alpha}(t)-\vec{r}_{j \beta}(0)\right]\right\}\right\rangle .
$$

Here $\vec{r}_{i \alpha}(t)\left[\vec{r}_{j \beta}(0)\right]$ is the position vector of the $i^{t h}$ atom of kind $\alpha\left[j^{t h}\right.$ atom of kind $\beta$ ] at time $=t$ [time $=0$ ] and the sum runs over all the different atoms of kinds $\alpha$ and $\beta\left[N_{\alpha}\left(N_{\beta}\right)\right.$ : total number of atoms of kind $\alpha(\beta)$; $\left.N=\sum_{\alpha} N_{\alpha}\right]$. Thus, $G_{\alpha \beta}(\vec{r}, t) d \vec{r}$ is the probability that, given a particle of kind $\beta$ at the origin at time $t=0$, any particle of kind $\alpha$ is in the volume $d \vec{r}$ at position $\vec{r}$ at time $t$. On the other hand, the self-part of the van Hove correlation function $G_{\alpha}^{\text {self }}(\vec{r}, t)$ is obtained by restricting the correlations considered in Eq. (A-1) to those relating the positions of a single particle of kind $\alpha$ at different times:

$$
G_{\alpha}^{\text {self }}(\vec{r}, t)=\left\langle\frac{1}{N} \sum_{i \alpha}^{N_{\alpha}} \delta\left\{\vec{r}-\left[\vec{r}_{i \alpha}(t)-\vec{r}_{i \alpha}(0)\right]\right\}\right\rangle .
$$

$G_{\alpha}^{\text {self }}(\vec{r}, t)$ is the Fourier transform of $S_{\alpha}^{\text {self }}(\vec{Q}, t)$ in space: Incoherent scattering relates to single particle motions.

The main equation in Fig. 10 shows that the weights of the coherent and incoherent contributions to the scattered intensity are determined by the scattering lengths of the isotopes involved. From Table 1 it is clear that:

- Due to the large value of $\overline{\Delta b_{H}^{2}}$, in H-containing systems the signal is dominated by the incoherent scattering from hydrogens, revealing their selfmotions. 


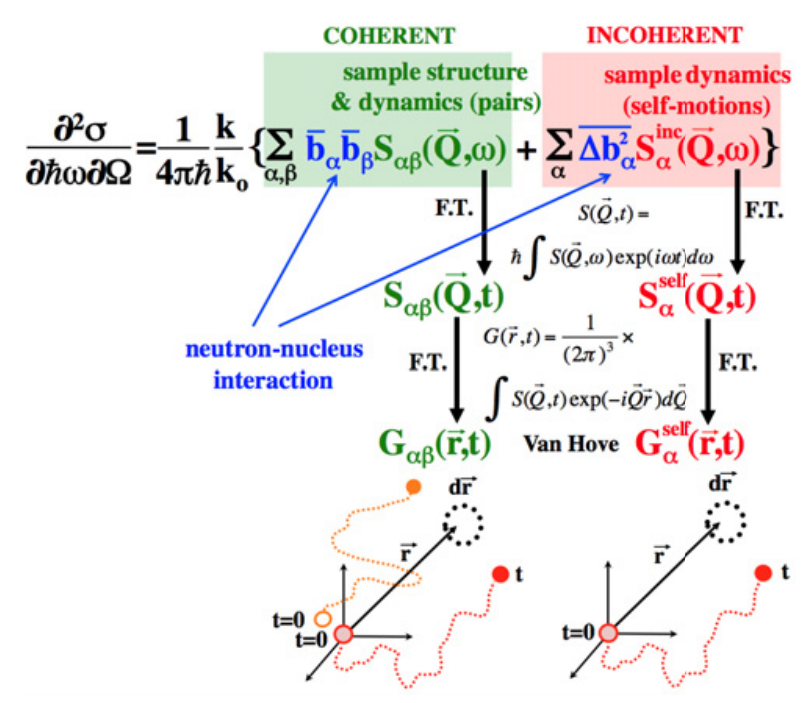

Figure 10. Scheme of the functions involved in the van Hove formalism of neutron scattering.

Table 1. Values of the average scattering lengths $\overline{b_{\alpha}}$, their squares ${\overline{b_{\alpha}}}^{2}$ and their deviations $\overline{\Delta b_{\alpha}^{2}}$ for different isotopes $\alpha$.

\begin{tabular}{lccc}
\hline Isotope $\alpha$ & $\overline{b_{\alpha}} / \mathrm{fm}$ & ${\overline{b_{\alpha}}}^{2} / \mathrm{fm}^{2}$ & $\overline{\Delta b_{\alpha}^{2}} / \mathrm{fm}^{2}$ \\
\hline${ }^{1} \mathrm{H}$ & -3.7406 & 13.992 & 638.78 \\
${ }^{2} \mathrm{H}(\mathrm{D})$ & 6.6710 & 44.502 & 16.322 \\
${ }^{12} \mathrm{C}$ & 6.6511 & 44.237 & 0 \\
${ }^{16} \mathrm{O}$ & 5.8030 & 33.675 & 0 \\
\hline
\end{tabular}

- Substituting H by D this incoherent contribution is drastically reduced and we obtain differently weighted coherent contributions.

- The intensity scattered by fully deuterated samples is mainly coherent and, since $\overline{b_{D}} \approx \overline{b_{C}}$, all pair correlations are almost equally weighted.

In practice, the intensities measured in experiments are affected through convolution by the instrumental resolution function $R(Q, \omega)$.

The Neutron Spin Echo (NSE) technique is unique since it determines the intermediate scattering functions directly in the time domain. This is achieved by coding the energy transfer in the scattering process for each neutron individually into its spin rotation [39]. In this way, the application of precession magnetic fields before and after the scattering event results in a polarization of the neutron that depends only on the velocity difference of each neutron individually, irrespective of its initial velocity. Energy resolution and monochromization of the incident beam are decoupled, and resolutions in energy of the order of $10^{-5}$ can be achieved with an incident neutron spectrum of $20 \%$ bandwidth. Moreover, as NSE works in the time domain, the deconvolution of the measured spectra from resolution can be realized by simple division of the data collected at the temperature of interest by the instrumental resolution spectrum. These experiments finally access the normalized function

$$
N S E(Q, t)=\frac{I_{c o h}(Q, t)-\frac{1}{3} I_{i n c}(Q, t)}{I_{c o h}(Q, 0)-\frac{1}{3} I_{i n c}(Q, 0)}
$$

where $I_{c o h}(Q, t)$ is the Fourier transform of the coherent double differential cross section $I_{c o h}(Q, t)=$ $\sum_{\alpha, \beta} \overline{b_{\alpha} b_{\beta}} S_{\alpha, \beta}(Q, t)$ and $I_{i n c}(Q, t)$ that of the incoherent double differential cross section $I_{\text {inc }}(Q, t)=$ $\sum_{\alpha} \overline{\Delta b_{\alpha}^{2}} S_{\alpha}^{\text {self }}(Q, t)$. From the above arguments it is followed that for fully deuterated samples, to a good approximation, the function measured by NSE can be identified with the normalized dynamic structure factor $S(Q, t) / S(Q)$-all atomic pair correlations are equally weighed- and for fully protonated samples, with the incoherent intermediate scattering function of the total hydrogens $S_{t H}^{\text {self }}(Q, t)$.

Neutron scattering techniques have some limitations, namely:

1. NS accesses correlation functions in the reciprocal space $(\vec{Q})$, never in real space.

2. The signals of different atoms, if they are of the same isotopic species, are not distinguished (e. g. main-chain hydrogens vs side-group hydrogens).

3. Self-motions of $\mathrm{C}$ and $\mathrm{O}$ are not accessible $\left(\overline{\Delta b_{C}^{2}}=\right.$ $\left.\overline{\Delta b_{O}^{2}}=0\right)$.

4. With exception of the neutron spin echo (NSE) technique (F. Mezei, 1972 [39]), that directly accesses the intermediate scattering functions, experiments are performed in the frequency domain and the results are affected by the instrumental resolution through convolution.

5. Spectrometers cover relatively narrow dynamic windows and usually several instruments have to be combined.

6. Though polarization analysis allows separation of coherent and incoherent contributions, in the practice this is currently not possible for the dynamic measurements.

Some of these limitations (4-6) might be overcome to a large extent with the development of the neutron sources and instrumentation, in particular with the spallation sources of new generation (JPARC, SNS and the future ESS). However, points (1-3) are inherent to the scattering processes.

\section{References}

[1] D. Richter, B. Frick, B. Farago, Phys. Rev. Lett. 61, 2465 (1988)

[2] R. Zorn, D. Richter, B. Farago, B. Frick, F. Kremer, U. Kirst, L.J. Fetters, Physica B 180\&181, 534 (1992)

[3] D. Richter, A. Arbe, J. Colmenero, M. Monkenbusch, B. Farago, R. Faust, Macromolecules 31, 1133 (1998)

[4] V. Arrighi, P. C., A. Triolo, S. Pouget, Physica B 301, 157 (2001)

[5] A. Arbe, A. Moral, A. Alegría, J. Colmenero, W. Pyckout-Hinzen, , D. Richter, B. Farago, B. Fick, J. Chem. Phys. 117, 1336 (2002) 
[6] B. Farago, A. Arbe, J. Colmenero, R. Faust, U. Buchenau, D. Richter, Phys. Rev. E 65, 051803 (2002)

[7] D. Richter, M. Monkenbusch, L. Willner, A. Arbe, J. Colmenero, B. Farago, Europhys. Lett. 66, 239 (2004)

[8] D. Richter, M. Monkenbusch, A. Arbe, J. Colmenero, in Neutron Spin Echo in Polymer Systems (Springer Verlag, Berlin Heidelberg New York, 2005), Vol. 174 of Advances in Polymer Science

[9] A.C. Genix, A. Arbe, F. Alvarez, J. Colmenero, B. Farago, A. Wischnewski, D. Richter, Macromolecules 39, 6260 (2006)

[10] A. Arbe, A.C. Genix, J. Colmenero, D. Richter, P. Fouquet, Soft Matter 4, 1792 (2008)

[11] M. Tyagi, A. Arbe, F. Alvarez, J. Colmenero, M.A. Gonzalez, J. Chem. Phys. 129, 224903 (2008)

[12] R. Pérez-Aparicio, A. Arbe, F. Alvarez, J. Colmenero, L. Willner, Macromolecules 42, 8271 (2009)

[13] M. Brodeck, F. Alvarez, A. Arbe, F. Juranyi, T. Unruh, O. Holderer, J. Colmenero, D. Richter, J. Chem. Phys. 130, 094908 (2009)

[14] V. García-Sakai, A. Arbe, Current Opinion in Colloid \& Interface Science 14, 381 (2009)

[15] J. Colmenero, A. Arbe, J. Polym. Sci.: Part B: Polym. Physics 51, 87 (2013)

[16] A. Arbe, F. Alvarez, J. Colmenero, Soft Matter 8, 8257 (2012)

[17] W. Götze, Complex Dynamics of Glass-Forming Liquids. A Mode Coupling Theory (Oxford University Press, New York, 2009)

[18] P.G. de Gennes, Physica 25, 825 (1959)

[19] K. Sköld, Phys. Rev. Lett. 19, 1023 (1967)

[20] J.P. Boon, S. Yip, Molecular Hydrodynamics (Dover publications, Mineola, NY, 1992)

[21] V.N. Novikov, K.S. Schweizer, A.P. Sokolov, J. Chem. Phys. 138, 164508 (2013)

[22] J. Colmenero, F. Alvarez, Y. Khairy, A. Arbe, J. Chem. Phys. 139, 044906 (2013)
[23] G.D. Patterson, J. Polym. Sci., Polym. Phys. Ed. 15, 455 (1977)

[24] R.S. Marvin, R. Aldrich, H.S. Sack, J. Appl. Phys. 25, 1213 (1954)

[25] M. Györ, Z. Fodor, H.C. Wang, R. Faust, Macromol. Sci., Pure Appl. Chem. A31, 2053 (1994)

[26] A. Narros, F. Alvarez, A. Arbe, J. Colmenero, D. Richter, B. Farago, J. Chem. Phys. 121, 3282 (2004)

[27] J. Colmenero, A. Alegría, A. Arbe, B. Frick, Phys. Rev. Lett. 69, 478 (1992)

[28] A. Arbe, J. Colmenero, F. Alvarez, M. Monkenbusch, D. Richter, B. Farago, B. Frick, Phys. Rev. Lett. 89, 245701 (2002)

[29] A. Arbe, J. Colmenero, F. Alvarez, M. Monkenbusch, D. Richter, B. Farago, B. Frick, Phys. Rev. E 67, 051802 (2003)

[30] T. Springer (Springer-Verlag, Berlin Heidelberg New York, 1972), Vol. 64 of Springer Tracts in Modern Physics

[31] P.A. Egelstaff, ed., An Introduction to the Liquid State (Oxford University Press, New York, 1992)

[32] Y. Khairy, F. Alvarez, A. Arbe, J. Colmenero, Macromolecules 47, 447 (2014)

[33] V.N. Novikov, Y. Ding, A.P. Sokolov, Phys. Rev. E 71, 061501 (2005)

[34] J. Colmenero, A. Arbe, A. Alegría, J. Non-Cryst. Solids 172-174, 126 (1994)

[35] S. Capponi, A. Arbe, S. Cerveny, R. Busselez, B. Frick, J. Embs, J. Colmenero, J. Chem. Phys. 134, 204906 (2011)

[36] S.W. Lovesey, Theory of Neutron Scattering from Condensed Matter (Clarendon Press, Oxford, 1984)

[37] M. Bée, Quasielastic Neutron Scattering (Adam Hilger, Bristol, 1988)

[38] G.L. Squires, Introduction to the Theory of Thermal Neutron Scattering (Dover Publication Inc., New York, 1996)

[39] F. Mezei, Neutron Spin Echo, Lecture Notes in Physics, Vol. 28 (Springer-Verlag, Heidelberg, 1980) 